PROCEEDINGS OF THE

AMERICAN MATHEMATICAL SOCIETY

Volume 126, Number 7, July 1998, Pages 2025-2032

S 0002-9939(98)04239-7

\title{
A GENERALIZATION OF CARLEMAN'S UNIQUENESS THEOREM AND A DISCRETE PHRAGMÉN-LINDELÖF THEOREM
}

\author{
B. KORENBLUM, A. MASCUILLI, AND J. PANARIELLO \\ (Communicated by Theodore W. Gamelin)
}

\begin{abstract}
Let $d \mu \geq 0$ be a Borel measure on $[0, \infty)$ and $A_{n}=\int_{0}^{\infty} t^{n} d \mu(t)<$ $\infty(n=0,1,2, \ldots)$ be its moments. T. Carleman found sharp conditions on the magnitude of $\left\{A_{n}\right\}_{0}^{\infty}$ for $d \mu$ to be uniquely determined by its moments. We show that the same conditions ensure a stronger property: if $A_{n}^{\prime}=\int_{0}^{\infty} t^{n} d \mu_{1}(t)$ are the moments of another measure, $d \mu_{1} \geq 0$, with limsup $\left|A_{n}-A_{n}^{\prime}\right|^{\frac{1}{n}}=\rho<$ $\infty$, then the measure $d \mu-d \mu_{1}$ is supported on the interval $[0, \rho]$. This result generalizes both the Carleman theorem and a theorem of J. Mikusiński. We also present an application of this result by establishing a discrete version of a Phragmén-Lindelöf theorem.
\end{abstract}

\section{$\S 1$. Preliminaries}

Definition 1.1. A sequence $\left\{A_{n}\right\}_{0}^{\infty}$ of positive numbers is called logarithmically convex if $A_{n} \leq \sqrt{A_{n-1} A_{n+1}}(n=1,2, \ldots)$.

Clearly, the moments $A_{n}=\int_{0}^{\infty} t^{n} d \mu(t)$ of any Borel measure $d \mu \geq 0$, with $A_{n}<$ $\infty$, form a logarithmically convex sequence.

Definition 1.2. If $\left\{A_{n}\right\}_{0}^{\infty}$ is an arbitrary sequence of positive numbers, then the convex regularization of $\left\{A_{n}\right\}_{0}^{\infty}$ by means of logarithms, denoted $\left\{A_{n}^{c}\right\}_{0}^{\infty}$, is formed by setting

$$
A_{n}^{c}=\sup \left\{B_{n}:\left\{B_{\nu}\right\}_{0}^{\infty} \text { is logarithmically convex, and } B_{\nu} \leq A_{\nu}(\nu=0,1, \ldots)\right\} .
$$

Proposition 1.3 ([Ma]). Given a sequence $\left\{A_{n}\right\}_{0}^{\infty}, A_{n}>0$, the following three conditions are equivalent:

Carleman's condition:

$$
\sum_{n=0}^{\infty} \frac{1}{\beta_{n}}=\infty, \quad \text { where } \quad \beta_{n}=\inf _{k \geq n} A_{k}^{\frac{1}{k}}
$$

Received by the editors June 13, 1996 and, in revised form, December 10, 1996.

1991 Mathematics Subject Classification. Primary 30E05; Secondary 26E10.

Key words and phrases. Carleman's uniqueness theorem, quasianalyticity, Phragmén-Lindelöf.

(C) 1998 American Mathematical Society 
Ostrowski's condition:

$$
\int_{1}^{\infty} \frac{\log T(r) d r}{r^{2}}=\infty, \quad \text { where } \quad T(r)=\sup _{n \geq 0} \frac{r^{n}}{A_{n}}
$$

Mandelbrojt's condition:

$$
\text { either } \quad \liminf _{n \rightarrow \infty} A_{n}^{\frac{1}{n}}<\infty \quad \text { or } \quad \sum_{n=0}^{\infty} \frac{A_{n}^{c}}{A_{n+1}^{c}}=\infty .
$$

We shall refer to the above as the (COM) condition.

Definition 1.4. We say a measure, $d \mu$, on $[0, \infty)$ is supported on $[0, \rho]$, if the total variation of $d \mu$ on $(\rho, \infty)$ is 0 , and $\rho$ is the smallest number having this property.

Theorem 1.5 ([C] Carleman's Uniqueness Theorem). If $A_{n}=\int_{0}^{\infty} t^{n} d \mu(t), d \mu \geq 0$, and $\left\{\sqrt{A_{n}}\right\}_{0}^{\infty}$ satisfies the $(C O M)$ condition, then no other measure $d \mu_{1} \geq 0$ has the same moments, $A_{n}$, for $n=0,1, \ldots$

Proposition 1.6 ([K] Analytic Quasianalyticity). Let $\left\{A_{n}\right\}_{0}^{\infty}$ be a sequence of positive numbers. Let $C\left\{A_{n}\right\}$ be the class of functions, $f(z)$, analytic in $\mathbb{D}=\{z \in \mathbb{C}$ : $|z|<1\}$ and infinitely differentiable on $\overline{\mathbb{D}}$ such that $\max _{z \in \mathbb{D}}\left|f^{(n)}(z)\right| \leq C_{f} A_{n}$. Then the following are equivalent:

The only $f \in C\left\{A_{n}\right\}$ vanishing with all of its

$$
\text { derivatives at a point } \zeta_{0} \in \partial \mathbb{D} \text { is } f(z) \equiv 0
$$

$$
\left\{\sqrt{A_{n}}\right\}_{0}^{\infty} \text { satisfies the }(C O M) \text { condition. }
$$

\section{$\S 2$. THE MAIN THEOREM}

Theorem 2.1. Let $d \mu \geq 0$ and $d \mu_{1} \geq 0$ be two Borel measures on $[0, \infty)$ with moments

$$
A_{n}=\int_{0}^{\infty} t^{n} d \mu(t) \quad \text { and } \quad A_{n}^{\prime}=\int_{0}^{\infty} t^{n} d \mu_{1}(t) \quad(n=0,1, \ldots) .
$$

If $\left\{\sqrt{A_{n}}\right\}_{0}^{\infty}$ satisfies the (COM) condition, and

$$
\limsup _{n \rightarrow \infty}\left|A_{n}-A_{n}^{\prime}\right|^{\frac{1}{n}}=\rho<\infty,
$$

then the measure $d \mu-d \mu_{1}$ is supported on $[0, \rho]$. Conversely, if $\left\{A_{n}\right\}_{0}^{\infty}$ is a sequence of positive numbers such that $\left\{\sqrt{A_{n}}\right\}_{0}^{\infty}$ does not satisfy the (COM) condition, then there are distinct measures $d \mu \geq 0$ and $d \mu_{1} \geq 0$ with

$$
\int_{0}^{\infty} t^{n} d \mu(t)=\int_{0}^{\infty} t^{n} d \mu_{1}(t) \leq A_{n} \quad(n=0,1, \ldots)
$$

Remark 2.2. The uniqueness case of the above theorem corresponds to $\rho=0$ :

$$
\lim _{n \rightarrow \infty}\left|A_{n}-A_{n}^{\prime}\right|^{\frac{1}{n}}=0 \text {. }
$$

The theorem then asserts that if $\left\{\sqrt{A_{n}}\right\}_{0}^{\infty}$ and $\left\{\sqrt{A_{n}^{\prime}}\right\}_{0}^{\infty}$ satisfy the (COM) condition, and (4) holds as well, then $A_{n}=A_{n}^{\prime}$ for $n=1,2, \ldots$ ( $A_{0} \neq A_{0}^{\prime}$ is possible). 
Remark 2.3. If we replace the hypothesis that $\left\{\sqrt{A_{n}}\right\}_{0}^{\infty}$ satisfies (COM) by the much stronger requirement

$$
\limsup _{n \rightarrow \infty} A_{n}^{\frac{1}{n}}<\infty
$$

then we obtain a theorem of Mikusiński [Mi].

Corollary 2.4. Let $d \mu$ be a signed Borel measure on $[0, \infty)$ with

$$
B_{n}=\int_{0}^{\infty} t^{n}|d \mu(t)|<\infty \quad(n=0,1, \ldots)
$$

$(|d \mu|$ is the variation measure of $\mu)$. If $\left\{\sqrt{B_{n}}\right\}_{0}^{\infty}$ satisfies the (COM) condition, then

$$
\rho=\limsup _{n \rightarrow \infty}\left|\int_{0}^{\infty} t^{n} d \mu(t)\right|^{\frac{1}{n}}=\limsup _{n \rightarrow \infty} B_{n}^{\frac{1}{n}}
$$

and $d \mu$ is supported on $[0, \rho](\rho=+\infty$ is not exluded $)$.

\section{§3. Proof of Theorem 2.1}

Define the signed measure, $d \sigma$, by $d \sigma=d \mu-d \mu_{1}$. We then define

$$
M_{n}=A_{n}-A_{n}^{\prime}=\int_{0}^{\infty} t^{n} d \sigma(t) .
$$

Also, let $B_{n}=\int_{0}^{\infty} t^{n}|d \sigma(t)| \leq A_{n}+A_{n}^{\prime}$. Define the function

$$
\Phi(z)=\int_{0}^{\infty} e^{t z} d \sigma(t) \quad \text { for } z \in \overline{\mathbb{C}_{-}}=\{z: \operatorname{Re}(z) \leq 0\} .
$$

$\Phi$ is easily seen to be analytic in $\mathbb{C}_{-}$, and differentiable in $\overline{\mathbb{C}_{-}}$, with

$$
\Phi^{(n)}(z)=\int_{0}^{\infty} t^{n} e^{t z} d \sigma(t) .
$$

We see that $\Phi^{(n)}(0)=M_{n}$ and $\left|\Phi^{(n)}(z)\right| \leq B_{n}$, for all $n=0,1,2, \ldots$ and for all $z \in \overline{\mathbb{C}_{-}}$.

Define another function, $F(z)$, via

$$
F(z)=\sum_{n=0}^{\infty} \frac{M_{n}}{n !} z^{n}
$$

$F$ is an entire function, and is of exponential type $\rho$ (i.e. $|F(z)| \leq C_{\epsilon} e^{(\rho+\epsilon)|z|}$ for all $\epsilon>0)$.

Now, we observe that $F^{(n)}(0)=\Phi^{(n)}(0)=M_{n}(n=0,1,2, \ldots)$. We know that $\left|\Phi^{(n)}(z)\right| \leq B_{n}$. Also,

$$
F^{(n)}(z)=\sum_{k=0}^{\infty} \frac{M_{n+k}}{k !} z^{k}
$$

and therefore

$$
\left|F^{(n)}(z)\right| \leq C_{R} R^{n}(n=0,1, \ldots) \quad \text { for all } R>\rho \text { and }|z| \leq 2 .
$$


Hence, we can conclude that on the closed disk $\{z:|z+1| \leq 1\}$,

$$
\left|\Phi^{(n)}(z)-F^{(n)}(z)\right| \leq B_{n}+C_{R} R^{n} \leq C_{R}^{\prime} B_{n} R^{n} .
$$

By Proposition 1.6, $\Phi-F \equiv 0$ on $\{z:|z+1| \leq 1\}$; that is, $F$ is an entire extension of $\Phi$.

We next let $F_{\delta}(z)=e^{-(\rho+\delta) z} F(z)$. This function is bounded on the imaginary axis (by $B_{0}$ ), bounded on the non-negative real axis (by a constant dependent on $\delta$ ) and of exponential type $\rho$. Hence, applying the Phragmén-Lindelöf theorem [Mar, vol. 2, p. 214], we can conclude that $\left|F_{\delta}(z)\right|$ is bounded on all of $\overline{\mathbb{C}_{+}}=\{z: \operatorname{Re}(z) \geq$ $0\}$ by $B_{0}$. Taking limits (as $\delta \rightarrow 0^{+}$), we see that

$$
|F(z)| \leq B_{0} e^{\rho \operatorname{Re}(z)} \quad \text { on } \overline{\mathbb{C}_{+}} .
$$

Define the function $G(z)$ by setting

$$
G(z)=\frac{F(z) e^{-\rho z}}{1+z}
$$

Clearly, $G$ is analytic on $\mathbb{C}_{+}$and is square summable on the imaginary axis. Thus, we can apply the Paley-Wiener theorem [PW, p. 8, Theorem V] to conclude that

$$
G(z)=\int_{-\infty}^{0} \Psi(t) e^{t z} d t \quad \text { for some } \Psi \in L^{2}((-\infty, 0))
$$

We will assume that $\Psi$ is defined for all real numbers (by setting $\Psi(t)=0$ for all $t>0$ ), and we will also extend our signed measure, $d \sigma$, to the entire real line (by requiring $|d \sigma((-\infty, 0))|=0)$.

On the imaginary axis, we have two representations for $G$ :

$$
\int_{-\infty}^{\infty} \Psi(t) e^{i y t} d t=G(i y)=\frac{e^{-i \rho y} F(i y)}{1+i y}
$$

So, we can conclude that

$$
\int_{-\infty}^{\infty} \Psi(t-\rho) e^{i y t} d t=\frac{1}{1+i y} \int_{-\infty}^{\infty} e^{i y t} d \sigma(t)
$$

Using the notation $\tilde{f}$ for the Fourier transform of $f$ (i.e. $\tilde{f}(x)=\int_{-\infty}^{\infty} f(y) e^{i y x} d y$ ), and letting $\Psi_{\rho}(t)=\Psi(t-\rho)$, we arrive at

$$
\widetilde{\Psi_{\rho}}(y)=\widetilde{\gamma}(y) \widetilde{d \sigma}(y)
$$

where $\gamma$ is the function

$$
\gamma(x)= \begin{cases}e^{x} & \text { if } x \leq 0 \\ 0 & \text { if } x>0\end{cases}
$$

Hence,

$$
\Psi_{\rho}(y)=(\gamma * d \sigma)(y)
$$


So

$$
\Psi(t-\rho)=\int_{t}^{\infty} e^{t-x} d \sigma(x)
$$

Thus, for all $t>\rho$, we have $\int_{t}^{\infty} e^{-x} d \sigma(x)=0$, which implies that the total variation of $d \sigma$ on $(\rho, \infty)$ is 0 ; i.e., $d \sigma=d \mu-d \mu_{1}$ is supported on $[0, \rho]$.

Conversely, if we are given a logarithmically convex sequence of positive numbers, $\left\{A_{n}\right\}_{0}^{\infty}$, with $\sum_{n=0}^{\infty} \sqrt{\frac{A_{n}}{A_{n+1}}}<\infty$, we set $A_{-1}=A_{0}$. A version of Proposition 1.6 for the half-plane $[\mathrm{K}]$ shows that there is some function, $f \not \equiv 0$, analytic in $\mathbb{C}_{+}$and continuous on $\overline{\mathbb{C}_{+}}$, with

$$
\sup _{x \geq 0} \int_{-\infty}^{\infty}\left|f^{(n)}(x+i y)\right|^{2} d y \leq A_{n-1}^{2} \quad(n=0,1,2, \ldots)
$$

and $f^{(n)}(0)=0(n=0,1,2, \ldots)$. Applying the same Paley-Wiener theorem, we obtain

$$
f(z)=\int_{0}^{\infty} \phi(t) e^{-t z} d t \quad \text { for some } \phi \in L^{2}((0, \infty))
$$

as well as

$$
f^{(n)}(z)=\int_{0}^{\infty} \phi(t)(-t)^{n} e^{-t z} d t
$$

Applying Plancherel's theorem, we find

$$
\int_{-\infty}^{\infty}\left|f^{(n)}(i y)\right|^{2} d y=2 \pi \int_{-\infty}^{\infty} t^{2 n}|\phi(t)|^{2} d t
$$

So, $\int_{0}^{\infty} t^{2 n}|\phi(t)|^{2} \leq A_{n-1}^{2}$. Hence, by the Cauchy-Schwarz inequality,

$$
\int_{0}^{\infty} t^{n}|\phi(t)| d t=\int_{0}^{1} t^{n}|\phi(t)| d t+\int_{1}^{\infty} t^{n+1}|\phi(t)| \frac{d t}{t} \leq A_{n-1}+A_{n} \leq K A_{n}
$$

for some constant $K$.

Letting $d \sigma=\frac{1}{K} \phi(t) d t$, and then defining $d \mu=d \sigma^{+}$and $d \mu_{1}=d \sigma^{-}$, we get the desired measures.

\section{$\S 4$. Some Applications}

In this section we examine some consequences of Theorem 2.1. The first result is a discrete Phragmén-Lindelöf type theorem.

Theorem 4.1. Let $f(z)$ be analytic in $\mathbb{C}_{+}=\{z: \operatorname{Re}(z)>0\}$ and continuous in $\overline{\mathbb{C}_{+}}$. Define

$$
A_{n}=\sup _{0 \leq x \leq n}|f(x+i y)| \quad \text { for } n=1,2, \ldots
$$

Assume $A_{n}<\infty$ for all $n$. 
If $\left\{\sqrt{A_{n}}\right\}_{0}^{\infty}$ satisfies the (COM) condition, and $\sup _{n \in \mathbb{N}}|f(n)|<\infty$, then $f(z)$ is bounded on $\overline{\mathbb{C}_{+}}$.

Proof. Consider the function

$$
g(z)=f(z)\left(\frac{1-e^{-z}}{z}\right)
$$

and let

$$
B_{n}=\sup _{0 \leq x \leq n}\left(\int_{-\infty}^{\infty}|g(x+i y)|^{2} d y\right)^{\frac{1}{2}} .
$$

We find that $B_{n} \leq 4 A_{n}$. Using the Paley-Wiener theorem for the strip [PW, p. 7, Theorem IV], we have

$$
g(x+i y)=\int_{-\infty}^{\infty} \phi(\sigma) e^{\sigma(x+i y)} d \sigma,
$$

where this integral converges for all $x>0$. Applying Plancherel's theorem yields

$$
\int_{-\infty}^{\infty}|g(n+i y)|^{2} d y=2 \pi \int_{-\infty}^{\infty} e^{2 n \sigma}|\phi(\sigma)|^{2} d \sigma \leq B_{n}^{2} \leq 16 A_{n}^{2}
$$

Setting $\Psi(t)=\phi(\log t)$ we get

$$
g(1+z)=\int_{0}^{\infty} t^{z} \Psi(t) d t \quad(\operatorname{Re} z>-1) .
$$

Also $(n \geq 0)$,

$$
\begin{aligned}
C_{n} & =\int_{0}^{\infty} t^{n}|\Psi(t)| d t=\int_{-\infty}^{\infty} e^{(n+1) t}|\phi(t)| d t \\
& =\int_{-\infty}^{0} e^{n t}|\phi(t)| e^{t} d t+\int_{0}^{\infty} e^{(n+2) t}|\phi(t)| e^{-t} d t \\
& \leq\left(\frac{1}{2} \int_{-\infty}^{0} e^{2 n t}|\phi(t)|^{2} d t\right)^{\frac{1}{2}}+\left(\frac{1}{2} \int_{0}^{\infty} e^{2(n+2) t}|\phi(t)|^{2} d t\right)^{\frac{1}{2}} \\
& \leq \frac{B_{n}+B_{n+2}}{2 \sqrt{\pi}} \leq \frac{4}{\sqrt{\pi}} A_{n+2} .
\end{aligned}
$$

Thus $\left\{\sqrt{C_{n}}\right\}_{0}^{\infty}$ also satisfies the $(\mathrm{COM})$ condition. Since $\sup _{n \in \mathbb{N}}|g(n)| \leq$ $\sup _{n \in \mathbb{N}}|f(n)|$, Corollary 2.4 yields $\Psi(t)=0(t>1)$ and $\phi(t)=0(t>0)$. Therefore

$$
f(z)=\frac{z}{1-e^{-z}} \int_{-\infty}^{0} \phi(\sigma) e^{\sigma z}
$$

which is bounded on every strip $\{0<\operatorname{Re} z<a\}$, satisfies $|f(z)|=O(|z|)(|z| \rightarrow \infty)$. Applying the Phragmén-Lindelöf theorem [Mar, vol. 2, p. 214], we see that $f$ is bounded on $\overline{\mathbb{C}_{+}}$.

Theorem 4.2. Let $f(z)$ be analytic in $\mathbb{C}_{+}$and continuous on $\overline{\mathbb{C}_{+}}$. Assume

$$
|f(i y)| \leq M \text { for all real } y \quad \text { and } \quad \sup _{n \in \mathbb{N}}|f(n)|<\infty \text {. }
$$


If $|f(z)| \leq C e^{p|z| \log |z|}$ for some $p<2$ and some constant $C$, then $|f(z)| \leq M$ for all $z \in \overline{\mathbb{C}_{+}}$.

Proof. Consider the function

$$
h_{1}(z)=f(z) e^{i \frac{p \pi}{2} z} z^{-p z}
$$

in the first quadrant: $Q_{1}=\left\{z: 0 \leq \operatorname{Arg}(z) \leq \frac{\pi}{2}\right\}$. This function is bounded on $\partial Q_{1}$, and in $Q_{1}$

$$
\left|h_{1}(z)\right|=O\left(e^{|z|^{1+\epsilon}}\right) \quad \text { for }|z| \rightarrow \infty, \epsilon>0 .
$$

Applying the same Phragmén-Lindelöf theorem [Mar] gives that $h_{1}(z)$ is bounded in $Q_{1}$. Using similar estimates on $h_{2}(z)=f(z) e^{-i \frac{p \pi}{2} z} z^{-p z}$ in the fourth quadrant $Q_{4}=\left\{z:-\frac{\pi}{2} \leq \operatorname{Arg}(z) \leq 0\right\}$ gives that $h_{2}(z)$ is bounded in $Q_{4}$. These estimates yield

$$
|f(x+i y)| \leq C \exp \left\{p x \log |x+i y|-(p y) \tan ^{-1}\left(\frac{y}{x}\right)+\frac{p}{2} \pi|y|\right\}
$$

in $\mathbb{C}_{+}$. Now, consider the function

$$
g(z)=f(z) e^{-\delta z^{\frac{p}{2}}} \quad \text { for any } \delta>0 .
$$

If

$$
D_{n}=\sup _{0 \leq x \leq n}|g(z)|
$$

then by a straightforward computation it is not hard to see that

$$
D_{n} \leq C_{\delta}^{n} e^{\pi n} n^{2 n} .
$$

So, in fact, the sequence $\left\{\sqrt{D_{n}}\right\}_{0}^{\infty}$ satisfies the (COM) condition. This implies that $|g(z)|$ is bounded in $\overline{\mathbb{C}_{+}}$by $M$. Letting $\delta \rightarrow 0^{+}$, we get

$$
|f(z)| \leq M \text { on } \overline{\mathbb{C}_{+}} \text {. }
$$

\section{ACKNOWLEDGEMENT}

The authors thank the referee for valuable criticism and suggestions.

\section{ADDED IN PROOF}

After the paper had gone to print, the authors learned that a result related to Theorem 2.1 is due to V. P. Havin and V. G. Maz'ya, Trudy Moskov. Mat. Obšč. 30 (1974), 61-114. MR 52:6000. The authors are grateful to Michael Sodin for this reference.

\section{REFERENCES}

[C] T. Carleman, Sur le Probléme des Moments, Comptus Rendus Acad. Sci. Paris 174 (1922), 1680 .

[K] B. Korenbljum, Quasianalytic Classes of Functions in a Circle, Soviet Mathematics (Doklady) 6 (1965), 1155-1158. MR 35:3074

[Ma] S. Mandelbrojt, Séries Adhérentes, Régularisation des Suites, Applications, GauthierVillars, Paris, 1952. MR 14:542f

[Mar] A. I. Markushevich, Theory of Functions of a Complex Variable, Chelsea Publishing Co., New York, 1977. MR 56:3258 
[Mi] J. Mikusiński, Remarks on the Moment Problem and on a Theorem of Picone, Colloquium Math. 2 (1951), 138-141. MR 13:214d

[PW] R. Paley and N. Wiener, Fourier Transforms in the Complex Domain, American Mathematical Society Colloquium Publications, vol. XIX, Providence, R.I., 1934. CMP 97:13

Department of Mathematics and Statistics, State University of New York at Albany, Albany, New York 12222 